\title{
Behaviour of a bentonite barrier in the laboratory: Experimental results up to 8 years and numerical simulation
}

\author{
M.V. Villar ${ }^{\mathrm{a}, *}$, M. Sánchez ${ }^{\mathrm{b}}, \mathrm{A}$. Gens $^{\mathrm{c}}$ \\ ${ }^{a}$ CIEMAT, Centro de Investigaciones Energéticas, Medioambientales y Tecnológicas, Avd. Complutense 22, 28040 Madrid, Spain \\ ${ }^{\mathrm{b}}$ University of Strathclyde, Department of Civil Engineering, John Anderson Building, 107 Rottenrow, Glasgow G40NG, United Kingdom \\ ${ }^{\mathrm{c}}$ UPC, Universitat Politècnica de Catalunya, Jordi Girona 1-3, 08034 Barcelona, Spain
}

\section{A R T I C L E I N F O}

\section{Article history}

Available online 14 October 2008

\section{Keywords:}

Bentonite

Engineered barrier

Laboratory tests

THM formulation

Modelling

\begin{abstract}
A B S T R A C T
The conditions of the bentonite in an engineered barrier for high-level radioactive waste disposal were simulated in a series of tests performed in cylindrical cells (length $60 \mathrm{~cm}$, diameter $7 \mathrm{~cm}$ ). Inside the cells, six blocks of FEBEX bentonite compacted to dry density $1.65 \mathrm{~g} / \mathrm{cm}^{3}$ were piled up, giving rise to a total length similar to the thickness of the clay barrier in a repository according to the Spanish concept. The bottom surface of the material was heated at $100^{\circ} \mathrm{C}$ and the top surface was injected with granitic water. The duration of the tests was $6,12,24$ and 92 months. The temperatures inside the clay and the water intake were measured during the tests and, at the end, the cells were dismounted and the dry density, water content and hydro-mechanical properties were measured at different positions. The injection of water provokes, near the hydration surface, a decrease of the dry density due to the increase of the water content and the clay swelling, while heating gives rise to an increase of the dry density and a reduction of the water content in the hottest areas.

A fully coupled thermo-hydro-mechanical (THM) formulation has been adopted as a general framework to analyse these experiments. This work presents the comparisons between the variables recorded online during the tests (water intake and temperature) and the model results. The main results of the postmortem analysis (water content and dry density) are also modelled.
\end{abstract}

(c) 2008 Elsevier Ltd. All rights reserved.

\section{Introduction}

The design of high-level radioactive waste (HLW) repositories in deep geological media includes the construction of a barrier around the waste containers constituted by a sealing material. Bentonite has been chosen as sealing material in most disposal concepts because of its low permeability, swelling capacity and retention properties, among other features. The behaviour of a HLW repository is determined, to a large extent, by the characteristics of the design and construction of the engineered barriers and especially by the changes that may occur in the mechanical, hydraulic, and geochemical properties as a result of the combined effects of heat generated by the radioactive decay and of the water and solutes supplied by the surrounding rock. Therefore, it is considered of fundamental importance for the evaluation of the longterm behaviour of the repository that the processes taking place in the near-field be understood and quantified.

\footnotetext{
* Corresponding author.

E-mail addresses: mv.villar@ciemat.es (M.V. Villar), marcelo.sanchez@strath. ac.uk (M. Sánchez), antonio.gens@upc.es (A. Gens).
}

Much attention has been paid since the 1980s to the performance of tests at different scales, in both the laboratory and the field, in order to observe the thermo-hydro-mechanical and geochemical (THM-G) processes taking place in the engineered barriers and the geological medium. The purpose of these experiments has been the direct observation of the phenomena occurring in the barrier and of the behaviour of the system, this providing the information required for the verification and validation of the mathematical models of the coupled processes and their numerical implementation.

The performance of large-scale in situ tests is complicated and time-consuming and the boundary conditions in them are not always well controlled. For this reason, laboratory tests at different scales in which the conditions of the bentonite in an engineered barrier for HLW disposal are simulated are very useful to identify and quantify processes.

The work presented here started in the framework of the EC FEBEX project, for the study of the near field for a HLW repository in crystalline rock according to the Spanish concept (ENRESA, 2006). The experimental work consisted of three main parts: (1) an in situ test, under natural conditions and at full scale (Grimsel, Switzerland); (2) a mock-up test, at almost full scale (CIEMAT, 
Madrid); and (3) a series of laboratory tests to complement the information from the two large-scale tests.

Among the laboratory tests started in the framework of the FEBEX Project - and continued in the NF-PRO Project - are those performed in cells in which the compacted bentonite is subjected simultaneously to heating and hydration, in opposite directions, in order to better understand the hydration process (Villar et al., 2005a, 2008). A series of infiltration tests performed under thermal gradient and dismantled after $0.5,1,2$ and 8 years operation are presented in this paper and the results obtained concerning water intake and final water content and density of the bentonite are analysed by means of a fully coupled THM formulation (Olivella et al., 1994).

The numerical analyses have been performed using the CODE_BRIGTH program (Olivella et al., 1996), which is a finite element code developed to handle coupled THM problems in porous media. The cells have been modelled as a boundary value problem. The geometry, initial conditions and boundary conditions have been adopted in order to reproduce as closely as possible the actual conditions of the tests. The modelling has considered the different stages of the tests, i.e. heating and hydration, cooling and dismantling (Villar et al,, 2005b). The extensive experimental campaign carried out during the FEBEX project has allowed to determine and estimate the main parameters of the constitutive models related to the thermal, mechanical and hydraulic problems.

\section{Theoretical formulation}

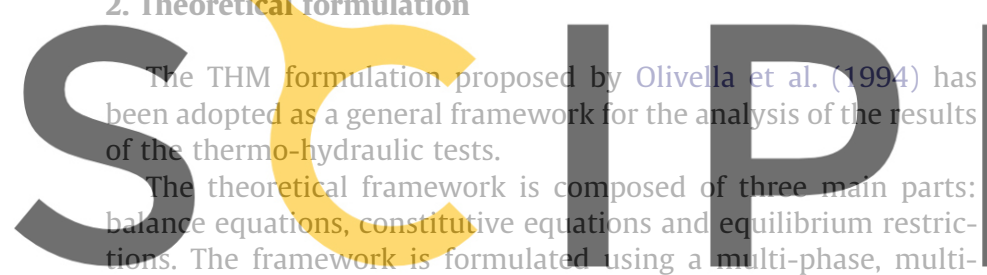
species approach. The subscripts identify the phase ('s' for solid, . 'l' for liguid and ' $g$ ' for gas), and the superscript indicates the spe-

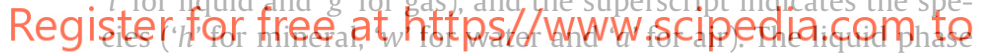
may contain water and dissolved air, and the gas phase may be a mixture of dry air and water vapour. Dry air is considered as single species. Thermal equilibrium between phases is assumed. This means that the three phases are at the same temperature.

\subsection{Balance equations}

Equations for mass balance were established following the compositional approach, which consists of balancing the species rather than the phases. The mass balance of solid present in the medium is written as

$\frac{\partial}{\partial t}\left(\rho_{s}(1-\phi)\right)+\nabla \cdot\left(\rho_{s}(1-\phi) \dot{u}\right)=0$

where $\rho_{s}$ is the mass of solid per unit volume of solid, $\phi$ is the porosity, $\mathbf{j}_{s}$ is the flux of solid, $t$ is time and $\nabla$ is the divergence operator.

Water is present in liquid and gas phases. The total mass balance of water is expressed as

$\frac{\partial}{\partial t}\left(\theta_{l}^{w} S_{l} \phi+\theta_{g}^{w} S_{g} \phi\right)+\nabla \cdot\left(\mathbf{j}_{l}^{w}+\mathbf{j}_{g}^{w}\right)=f^{w}$

where $\theta_{l}^{w}$ and $\theta_{g}^{w}$ are the masses of water per unit volume of liquid an gas respectively; $S_{l}$, is the volumetric fraction of pore volume, occupied by the alpha phase $(\alpha=l, g) ; \mathbf{j}_{l}^{w}$ and $\mathbf{j}_{g}^{w}$ denote the total mass fluxes of water in the liquid and gas phases (water vapour) with respect to a fixed reference system and $f^{w}$ is an external supply of water.

Dry air is present in liquid and gas phases. The total mass balance of dry air is expressed as $\frac{\partial}{\partial t}\left(\theta_{l}^{w} S_{l} \phi+\theta_{g}^{w} S_{g} \phi\right)+\nabla \cdot\left(\mathbf{j}_{l}^{w}+\mathbf{j}_{g}^{w}\right)=f^{w}$

where $\theta_{l}^{a}$ and $\theta_{g}^{a}$ are the masses of dry air per unit volume of liquid an gas, respectively; $S_{\alpha}$ is the volumetric fraction of pore volume, occupied by the alpha phase $(\alpha=l, g) ; \mathbf{j}_{l}^{a}$ and $\mathbf{j}_{g}^{a}$ denote the total mass fluxes of dry air in the liquid and gas phases with respect to a fixed reference system and $f^{a}$ is an external supply of dry air.

Regarding the thermal problem, equilibrium between the phases is assumed. Therefore, the temperature is the same for all the phases. Consequently, only one equation is needed for the energy balance. The total internal energy, per unit volume of porous media, is obtained adding the internal energy of each phase corresponding to each medium. Applying the balance equation to this quantity, the following equation is obtained:

$$
\begin{aligned}
& \frac{\partial}{\partial t}\left(E_{S} \rho_{s}(1-\phi)+E_{l} \rho_{l p} S_{l} \phi+E_{g} \rho_{g} S_{g} \phi\right)+\nabla \cdot\left(\mathbf{i}_{c}+\mathbf{j}_{E S}+\mathbf{j}_{E l}+\mathbf{j}_{E g}\right) \\
& \quad=f^{E}
\end{aligned}
$$

where $E_{S}$ is the solid specific internal energy; $E_{l}$ and $E_{g}$ are specific internal energies corresponding to liquid and gas phase, respectively, $\rho_{S}$ is the solid density; $\rho_{l}$ and $\rho_{g}$ are the liquid and gas phase densities; $\mathbf{i}_{C}$ is the conductive heat flux; $\mathbf{j}_{E S}$ is the advective energy flux of solid phase with respect to a fixed reference system; $\mathbf{j}_{E I}$ and $\mathbf{j}_{\text {Eg }}$ are the advective energy flux of liquid and gas phases, respectively, with respect to a fixed reference system; $f^{E}$ are the energies supply per unit volume of medium.

Finally, the balance of momentum for the porous medium re-

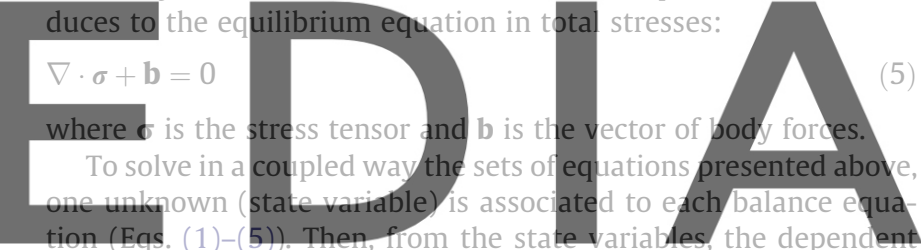

variables are calculated using the constitutive equations (Section

download the version without the watermark

\subsection{Constitutive equations}

The constitutive equations establish the link between the independent variables (or unknowns) and the dependent variables. In this section some constitutive laws will be introduced in a generic way, and the particular values of the parameters are given in Section 3. A more detailed explanation of the mathematical formulation in general and constitutive equations in particular is given in Gens et al. (1998). Concerning the hydraulic problem it is assumed that the liquid and gas flows follow Darcy's law:

$\mathbf{q}_{\alpha}=-\mathbf{K}_{\alpha}\left(\nabla P_{\alpha}-\rho_{\alpha} \mathbf{g}\right) ; \quad \alpha=l, g$

where $P_{l}$ and $P_{g}$ are liquid and gas pressure, respectively, $\rho_{l}$ is the liquid density, $\rho_{g}$ is the gas density, $\mathbf{g}$ is the gravity vector and $\mathbf{K}_{\alpha}$ is the permeability tensor of the alpha phase $(\alpha=l, g)$, which is given by

$\mathbf{K}_{\alpha}=\mathbf{k} \frac{k_{r, \alpha}}{\mu_{\alpha}} ; \quad \alpha=l, g$

The intrinsic permeability tensor ( $\mathbf{k})$ depends on the pore structure of the porous medium. $k_{r \alpha}$ is the value of relative permeability that controls the variation of permeability in the unsaturated regime and $\mu_{\alpha}$ denotes the dynamic viscosity. $\alpha$ may stand for either $l$ or $g$ depending on whether liquid or gas flow is considered. The relative permeability of liquid $\left(k_{r l}\right)$ phases is made dependent on $S_{e}$ (effective degree of saturation) according to

$k_{r l}=S_{e}^{n} \quad(\mathrm{a}) ; \quad S_{e}=\frac{S_{l}-S_{l r}}{S_{l s}-S_{l r}}$

(b) 
where $S_{l}$ is degree of saturation, $S_{l r}$ and $S_{l s}$ are residual and maximum degree of saturation, respectively, and $n$ is a material parameter. Another relevant law related to the hydraulic problem is the retention curve.

The molecular diffusion of vapour water in gas phase is governed by Fick's law, through

$\mathbf{i}_{g}^{w}=-\mathbf{D}_{g}^{w} \nabla \omega_{g}^{w}=-\left(\phi \rho_{g} S_{g} \tau D_{m}^{w} \mathbf{I}+\rho_{g} \mathbf{D}_{g}^{\prime}\right) \nabla \omega_{g}^{w}$

where $\mathbf{i}_{w}^{g}$ is the non-advective mass flux of water in gas, $\mathbf{D}_{w}^{g}$ is the dispersion tensor, $\omega_{w}^{g}$ is the mass fraction of water in gas, $\tau$ is the tortuosity and $\mathbf{D}_{g}^{\prime}$ the mechanical dispersion tensor. $\mathbf{D}_{w}^{m}$ is the molecular diffusion coefficient of vapour in gas.

As for the thermal problem, the Fourier's law has been adopted for the conductive flux of heat. Thermal conductivity depends on the hydration state of the clay and is expressed by a variant of the geometric mean:

$\mathbf{i}_{c}=-\lambda \nabla T$
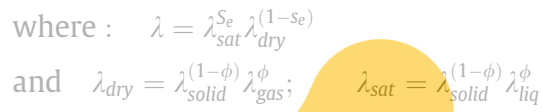

\section{where $\lambda$ is the thermal conductivity}

Finally, regarding the mechanical problem, the constitutive model known as "BBM" (Barcelona Basic Model) has been adopted (see Appendix A). Due to the high compaction the bentonite blocks have been subjected to, the description of the behaviour of the material inside the yield surface is particularly important. The variation of stress-stiffness with suction and, especially, the varigtion
of swelling potential with stress and suction have been considered
(Sanchez and Gens, 2006). The resulting elastic model is the
following:
$\epsilon_{v}^{e}=\frac{\kappa}{(1+e)} \frac{\dot{p}}{p}+\frac{\kappa_{s}}{(1+e)} \frac{s}{(s+0.1)}+\left(\alpha_{d}+\alpha_{2} \Delta T\right) \dot{T}$ $\dot{\epsilon}_{s_{0}}^{e}=\frac{\dot{J}}{C}$

$\kappa_{s}=\kappa_{s 0}\left(1+\alpha_{s p} \ln p / p_{\text {ref }}\right)$

$G=\frac{E}{2(1+v)}$

where $E$ is the young module.

\subsection{Equilibrium restrictions}

Other types of relationships that relate dependent variables with unknowns are the equilibrium restrictions. They are obtained assuming chemical equilibrium for dissolution of the different species (dry gas and vapour) in phases (liquid, gas). This assumption is sufficiently adequate because these chemical processes are fast compared to the transport processes that take place in porous media and, for this reason; they are not rate-controlled. In this problem the concentration of water vapour in the gas phase is controlled by the psycrhometric law, and the solubility of dry gas in water is given by Henry's law (Olivella et al., 1994).

\section{Material}

The cell tests have been performed with the FEBEX bentonite, selected by ENRESA (the Spanish Agency for Radioactive Waste Management) as suitable material for the backfilling and sealing of HLW repositories. The bentonite is extracted from the Cortijo de Archidona deposit (Almería, Spain). Processing of the material at the factory consisted in disaggregation and gently grinding, dry- ing at $60{ }^{\circ} \mathrm{C}$ and sieving by $5 \mathrm{~mm}$. For the large-scale tests of the Project, the bentonite blocks were manufactured by uniaxial compaction of the granulated clay with its hygroscopic water content, at dry densities close to $1.7 \mathrm{~g} / \mathrm{cm}^{3}$ (ENRESA, 2006).

The physico-chemical properties of the FEBEX bentonite, as well as its most relevant thermo-hydro-mechanical and geochemical characteristics are summarised in the final reports of the project (ENRESA, 2006). Several laboratories participated in these characterisation tasks. A summary of the results obtained is given below.

The FEBEX bentonite has a content of montmorillonite higher than $90 \%$ with variable quantities of quartz, plagioclase, K-feldspar, calcite and opal-CT (cristobalite-trydimite). The cation exchange capacity (CEC) varies from 96 to $102 \mathrm{meq} / 100 \mathrm{~g}$. Major exchangeable cations are: Ca (35-42 meq/100 g), Mg (31-32 meq/100 g), $\mathrm{Na}(24-27 \mathrm{meq} / 100 \mathrm{~g})$ and $\mathrm{K}(2-3 \mathrm{meq} / 100 \mathrm{~g})$.

The liquid limit of the bentonite is $102 \pm 4 \%$, the plastic limit is $53 \pm 3 \%$, the specific gravity $2.70 \pm 0.04$, and $67 \pm 3 \%$ of particles are smaller than $2 \mu \mathrm{m}$. The hygroscopic water content in equilibrium with the laboratory atmosphere is $13.7 \pm 1.3 \%$. The external specific surface area, using the BET technique, is $32 \pm 3 \mathrm{~m}^{2} / \mathrm{g}$ and the total specific surface area, using the hygroscopicity method, is about $725 \mathrm{~m}^{2} / \mathrm{g}$. The analysis of the mercury intrusion data reveals that the intra-aggregate pores (smaller than $0.006 \mu \mathrm{m}$ ) represents the $73-78 \%$ of total pore volume when the bentonite is compacted at a dry density of $1.7 \mathrm{~g} / \mathrm{cm}^{3}$.

The saturated permeability to deionised water, k (Eq. (7)), of samples of untreated FEBEX bentonite compacted at different dry densities can be related with perosity through a modified Kozeny's
law according to
$\mathbf{k}=k_{0} \frac{\phi^{3}}{(1-\phi)^{2}} \frac{\left(1-\phi_{0}\right)^{2}}{\phi_{0}^{3}}$ I
where $k_{0}$ is the intrinsic permeability corresponding to $\phi_{0}$ (a refer-
ence porosity).

Fig. 1 presents the law adopted for FEBEX bentonite permeated

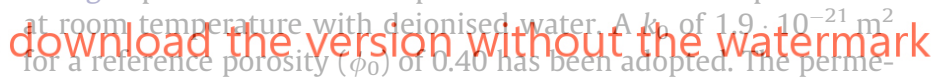
ability of the FEBEX bentonite to granitic water (salinity $0.02 \%$ ) is analogous to that obtained for deionised water (Villar, 2002).

The retention curve of the bentonite was determined in samples compacted to different dry densities (Lloret et al., 2004). The volume of the samples remained constant during the determinations, since they were confined in constant volume cells. To impose the different relative humidities the cells were placed in desiccators with sulphuric acid solutions of various concentrations. A modified

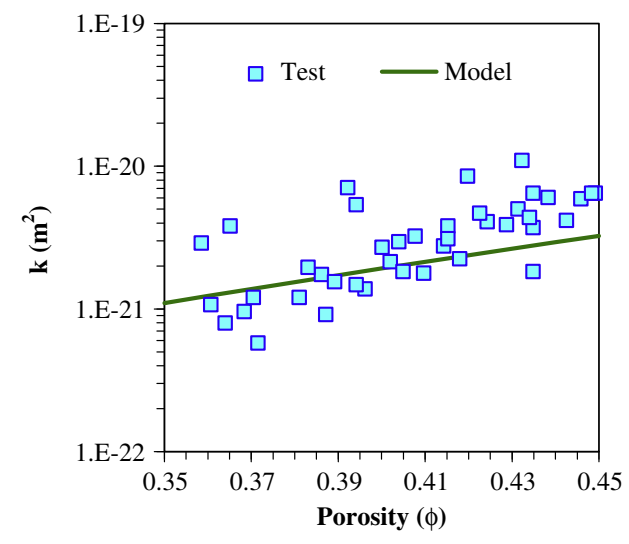

Fig. 1. Variation of saturated permeability with porosity. Experimental data and adopted model for the intrinsic permeability law. 
van Genuchten law has been adopted to model the dependence of the degree of saturation on suction

$S_{e}=\left[1+\left(\frac{s}{P_{0}}\right)^{\frac{1}{1-\lambda_{0}}}\right]^{-\lambda_{0}} f_{d} ; \quad$ where $: f_{d}=\left(1-\frac{s}{P_{d}}\right)^{\lambda_{d}}$

where $s\left(s=P_{g}-P_{l}\right)$, is the suction, $P_{0}$ is a parameter related to the capillary pressure (the air entry value) and $\lambda_{0}$ is a parameter that controls the shape of the curve (van Genuchten, 1978). The function $f_{d}$ is included to obtain more suitable values at high suctions, $P_{d}$ is a parameter related with the suction at 0 degree of saturation and $\lambda_{d}$ is a model parameter (when it is null, the original model is recovered). Fig. 2 presents the retention curves obtained with these expressions for different values of dry density together with the experimental data. The adopted parameters are: $P_{0}=28 \mathrm{MPa}$, $\lambda_{0}=0.18, P_{d}=1100 \mathrm{MPa}, \lambda_{d}=1.1$ and $\eta=0.7$.

The relation between $P_{0}$ and surface tension $(\sigma)$ suggested by Olivella and Gens (2000) has been extended to this model, that is

$P_{0}=P_{T_{0}} \frac{\sigma_{T}}{\sigma_{T_{0}}}$

where the surface tension (in $\mathrm{N} / \mathrm{m}$ ) has been obtained fitting values of surface tension with the following expression (Olivella, 1995):

$\sigma_{T}=0.03059 \exp \left(\frac{252.93}{273.15+T}\right)$

By varying $P_{0}$ in accordance with this expression, a dependence of
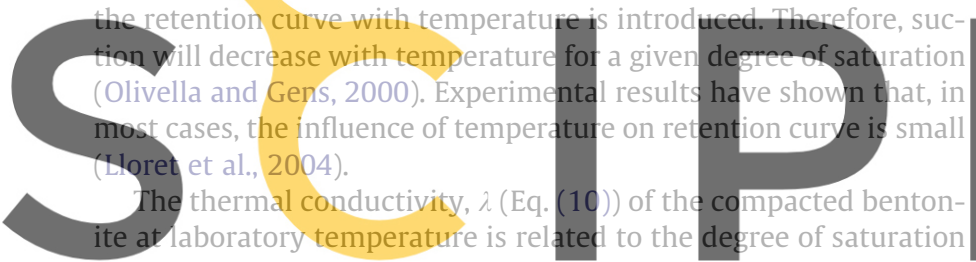

$S_{e}(E q .(11))$. Fig. 3 presents the experimental values obtained for

the FEBEX bentonite together with the model result computed

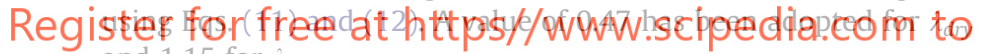
and 1.15 for $\lambda_{\text {sat }}$

Some isothermal infiltration tests and heat flow tests at constant overall vater content vere performed during FEBEX $I$ and they were back-analysed using CODEBRIGHT (Pintado et al., 2002). It is possible to fit the experimental data for the relative permeability law (Eq. (8)) assuming a cubic law (i.e. $n=3$ ). As for the tortuosity factor, $\tau$ (Eq. (9)), a value of 0.8 has been proposed.

Regarding the mechanical properties, the THM version of the BBM presented in Appendix A has been adopted in this analysis. However, the BBM being not able to reproduce the swelling behav-

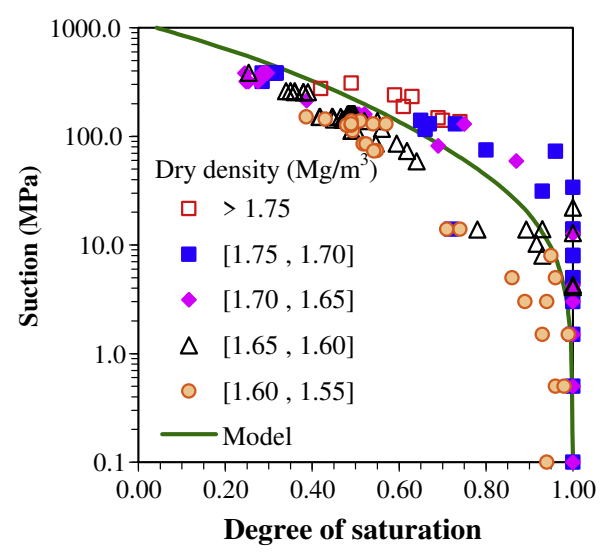

Fig. 2. Retention curve adopted in the analyses, together with the experimental data for FEBEX bentonite (symbols).

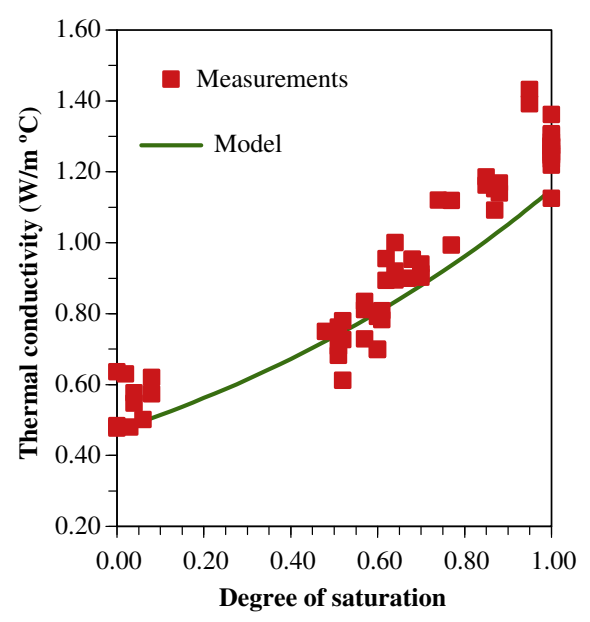

Fig. 3. Thermal conductivity: FEBEX bentonite experimental results (symbols) and model fitting,

iour of the bentonite, a number of modifications have been introduced in the elastic part of the model in order to reproduce the expansive behaviour of the FEBEX bentonite. Due to the high pressures applied to compact the bentonite blocks, the description of the behaviour of the material inside the yield surface is particularly important. The variation of stress-stiffness with suction and, espe-

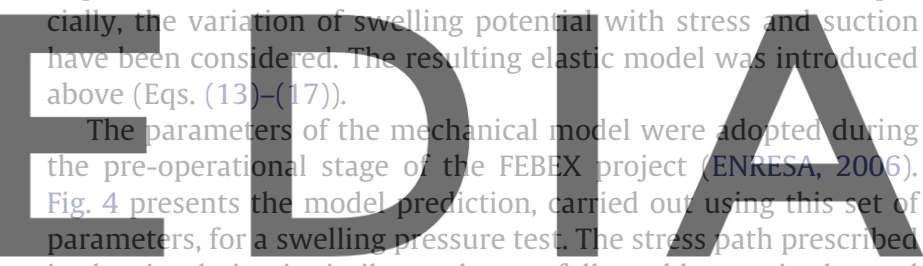

in the simulation is similar to the one followed by a point located close to the heater, that is, initial drying (in this case close to a suc-

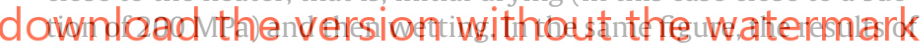
the swelling pressure tests coded as SP1 and SP2, described in Lloret et al. (2003), are shown. Note that the model results can be considered as modei predictions, because these tests were carried out after parameter determination. As it can be observed, the model results can be considered satisfactory, because the stress path is quite well reproduced as well as the predicted value of the swelling pressure. The main model parameters are summarised in Table 1. More details can be found in Sánchez and Gens (2006).

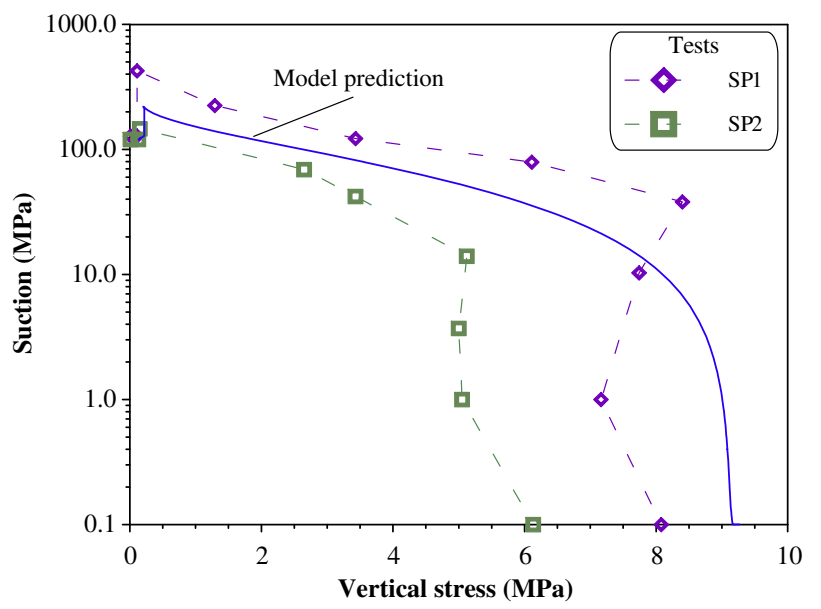

Fig. 4. Computed stress path for swelling pressure tests using the BBM. SP1 and SP2 experimental results are provided for comparison. 
Table 1

Model parameters related to the mechanical problem (defining the Barcelona Basic Model).

\begin{tabular}{llll}
\hline$\kappa$ & 0.04 & $\kappa_{s}$ & 0.25 \\
$\nu$ & 0.4 & $\alpha_{i s}$ & -0.003 \\
$\alpha_{s p}$ & -0.147 & $\alpha_{s s}$ & 0.00 \\
$\alpha_{0}$ & $1.5 \times 10^{-4}\left({ }^{\circ} \mathrm{C}^{-1}\right)$ & $\alpha_{2}$ & 0.00 \\
$\lambda_{0_{*}}$ & 0.15 & $p_{c}$ & $0.10 \mathrm{MPa}$ \\
$p_{0}$ & $14 \mathrm{MPa}$ & $\alpha$ & 0.395 \\
$r$ & 0.75 & $\beta$ & 0.05 \\
$M$ & 1.5 & $T_{\mathrm{o}}$ & $20^{\circ} \mathrm{C}$ \\
$k$ & 0.1 & $\rho$ & 0.2 \\
\hline
\end{tabular}

\section{Methods}

\subsection{Thermo-hydraulic tests}

A series of infiltration tests in large-scale cells (inner length $60 \mathrm{~cm}$, internal diameter $7 \mathrm{~cm}$ ) was running for different periods of time ( 0.5 to 7.6 years). The cells were made of Teflon to prevent lateral heat conduction, and externally covered with steel semicylindrical pieces to avoid deformation of the cell by bentonite swelling. Six $10 \mathrm{~cm}$ height blocks of FEBEX clay compacted with its hygroscopic water content at an initial nominal dry density of $1.65 \mathrm{~g} / \mathrm{cm}^{3}$, were piled up inside each cell. The actual average initial water content of the bentonite in the seven tests performed



Hydration with granitic water (salinity 0.02\%) took place through the upper surface under an injection pressure of 1.2 MPa. The water intake was measured as a function of time in some of the tests with electronic equipment placed at the entrance of the cell (LVDT displacement transducer that allows measuring changes of volume with an accuracy of $0.001 \mathrm{~cm}^{3}$ ).

In addition, the cells were instrumented with thermocouples inserted in the bentonite at different levels along the column. The number of thermocouples in each cell was 2,3 or 5 . Only the 7.6-year duration test was not instrumented with thermocouples (Fig. 5).

Seven tests were performed: two of 0.5 year duration, two of 1 year duration, two of 2 years duration and one of 7.6 years duration. Of the duplicate tests, the FQ ones were used for postmortem determination of hydro-mechanical properties, and the HI ones for postmortem determination of geochemistry and extraction of interstitial water. The longest test, CG3, was used for both kinds of nostmortem determinations.

\subsection{Postmortem analysis}

At the end of the thermo-hydraulic treatment, the cells were dismantled and the clay blocks extracted. Special care was taken to avoid any disturbance in the conditions of the clay, particularly its dry density and water content. Once extracted, the bentonite columns were sawed in 24 cylindrical sections of $2.5 \mathrm{~cm}$ in thickness (numbered from 1, near the hydration surface, to 24, close

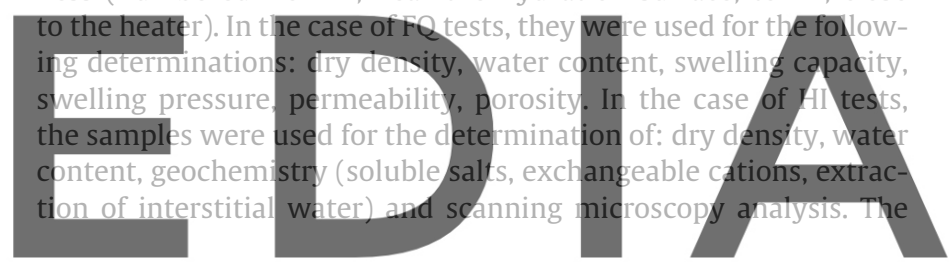

Register for free at https//www.scipedia.com to download the version without the watermark
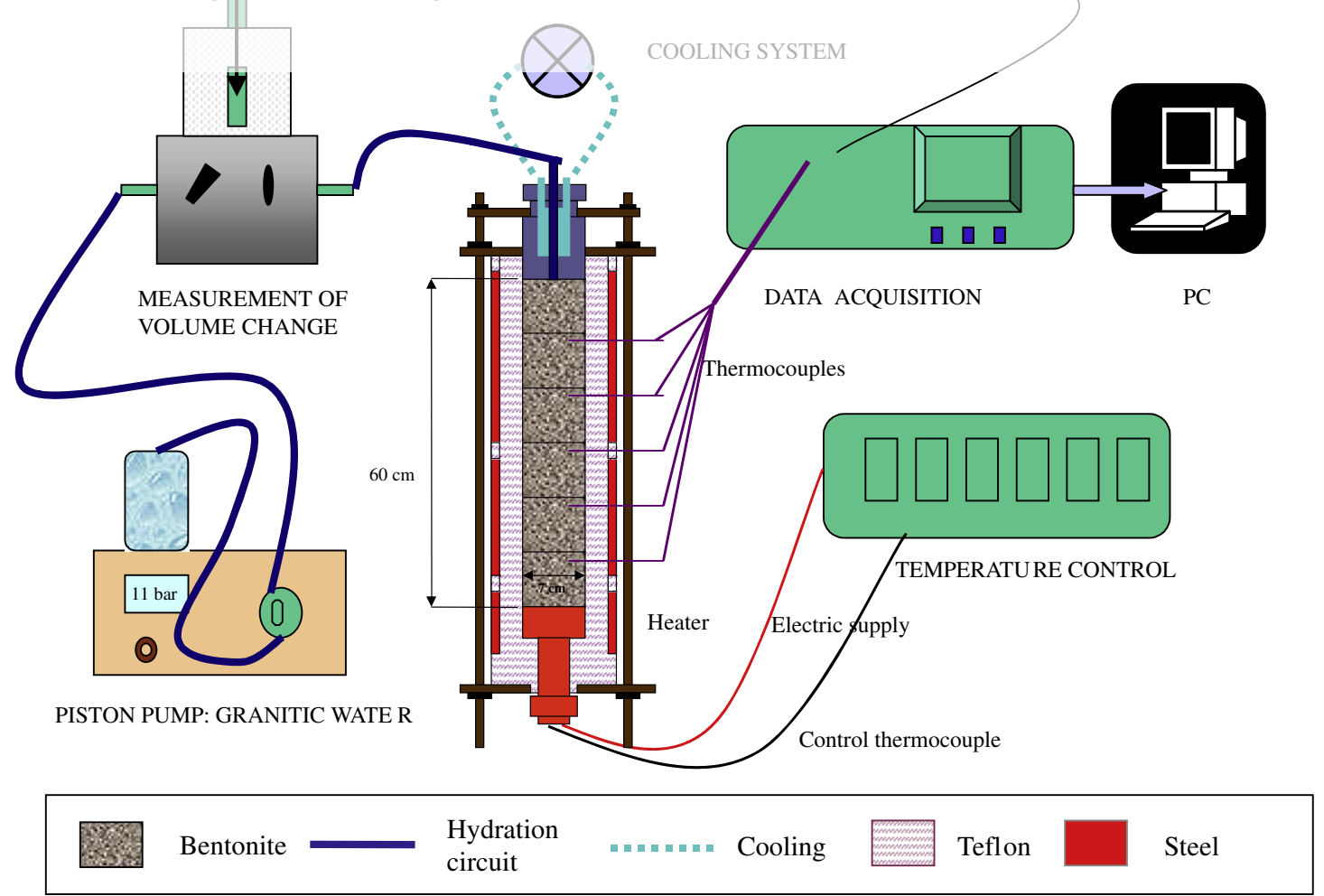

Fig. 5. Experimental setup for the infiltration tests (Villar et al., 2005). 
samples from test CG3 were used for determination of dry density, water content, swelling capacity, specific surface area, mineralogy and geochemistry. This paper analyses only the results on final dry density and water content.

The gravimetric water content $(w)$ was determined by oven drying at $110^{\circ} \mathrm{C}$ for 24 hours (defined as the ratio between the weight of water and the weight of dry solid). Dry density $\left(\rho_{d}\right)$ is defined as the ratio between the weight of the dry sample and the volume occupied by it prior to drying. The volume of the specimens was determined by immersing them in a recipient containing mercury and by weighing the mercury displaced, as established in UNE Standard 7045 "Determination of soil porosity."

\subsection{Modelling}

The finite element program CODE_BRIGHT (Olivella et al., 1996) has been used to analyze the cells as a boundary value problem. 1D axis-symmetrical models have been used in these analyses. A mesh of one hundred (100) elements has been chosen. A sensitivity analysis has also been carried out to verify that the model results do not depend on the mesh. The initial and boundary conditions of the model have been imposed in order to be the closest possible to the experiments. The initial water content of the bentonite block is close to $14 \%$, from the retention curve adopted an initial value of suction close to $140 \mathrm{MPa}$ has been chosen. An initially uniform temperature of $22{ }^{\circ} \mathrm{C}$ is assumed. Initial hydrostatic stresses of $0.15 \mathrm{MPa}$ have been adopted. Regarding the boundary conditions a temperature of $100^{\circ} \mathrm{C}$ is imposed at and bentonite (the bottom of the ce sure of $1.2 \mathrm{MPa}$ is imposed at the o part) The thermal boundary conditior acopted in order to adjust the ten temperature of $23^{\circ} \mathrm{C}$ has been fix of 1 (one). Regarding the mechanical problent, a small expansion of the cell has been allowed, in order to account for the deformability of the Teflon walls upon saturation and swelling of the benton-

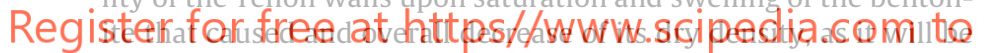
shown in Section 5.2. Finally, a constant gas pressure (0.1 MPa) has been adopted in the analyses.

\section{Experimental and numerical results}

\subsection{Online measurements}

The initiation of the thermo-hydraulic tests followed this procedure: first the temperature of the upper cooling circuit was set to $30^{\circ} \mathrm{C}$, after $24-48 \mathrm{~h}$ the temperature of the bottom heater was set to $100^{\circ} \mathrm{C}$ and hydration started almost simultaneously (except in tests FQ1/2, HI1/2 and FQ2, in which hydration started $24 \mathrm{~h}$ afterwards). During the hydration-heating tests temperature measurements in different positions along the columns and water intake measurements (only in four of the tests) were performed.

During the $\mathrm{TH}$ tests, temperature was measured at different positions along the columns. In test CG3 no thermocouples were inserted. The temperatures quickly stabilised (in less than $200 \mathrm{~h}$ ) and afterwards, they were mainly affected by the room temperature, reflecting the daily and seasonal changes, especially in the zones farther from the heater (Fig. 6). The average temperatures recorded by each thermocouple for the period between $700 \mathrm{~h}$ after the beginning of the tests and the end of the tests are plotted in Fig. 7. There are is a good agreement between the average temperatures measured in the duplicate tests of the same duration. There is a sharp temperature gradient in the vicinity of the heater, decreasing from $100^{\circ} \mathrm{C}$ at the heater surface to $50^{\circ} \mathrm{C}, 10 \mathrm{~cm}$ inside the clay. In addition, a decrease of temperature with saturation

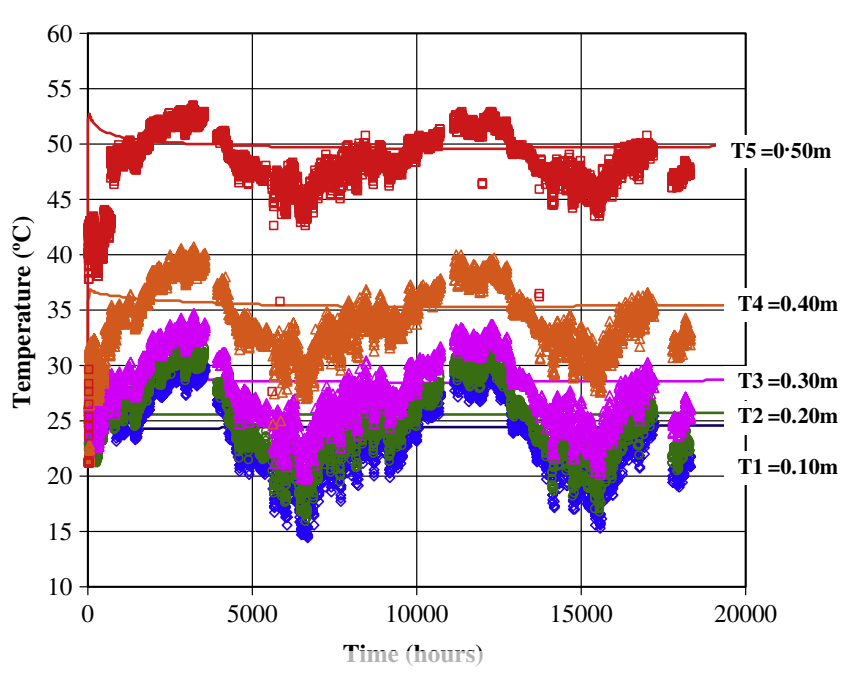

Fig. 6. Temperatures recorded by thermocouples in test HI2 (T1 to T5 were placed at $50,40,30,20$ and $10 \mathrm{~cm}$ from the heater, respectively): experimental and modelling results.

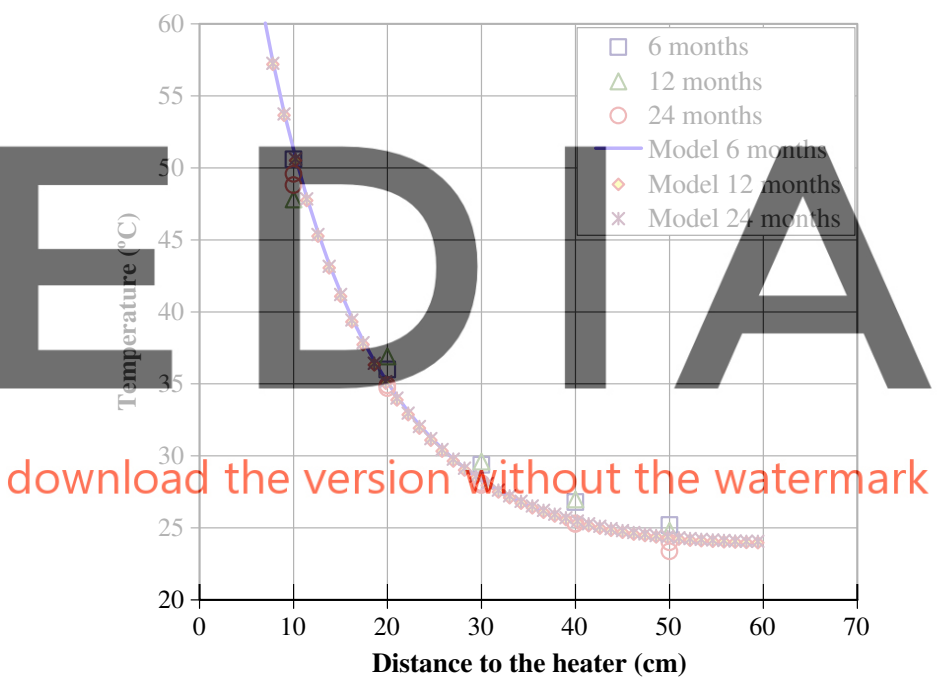

Fig. 7. Average steady temperatures at three different positions inside the clay for tests of different duration: experimental and modelling results.

(longest tests) is observed, especially in the wettest zones, i.e. within the closest $40 \mathrm{~cm}$ to the hydration surface. This is due to the higher heat dissipation of the wet clay.

Since the model only intends to capture the whole thermal evolution of the test, the cyclic variations of temperature (due to the changes in the laboratory temperature) are not taken into account. Considering that, it can be seen that the model captures quite well the thermal field prevailing in the cell (Figs. 6 and 7).

The longest test, test CG3, had no thermocouples inserted in the clay, but before switching off the heater, the temperatures on the Teflon surface of the cell were measured with a hand-held thermistor thermometer. Although the external temperatures were lower than those inside the bentonite, a higher thermal gradient was observed in the proximity of the heater, and a soft decrease in the farthest area, where the temperature was very close to the room temperature at the moment of dismantling $\left(22^{\circ} \mathrm{C}\right)$.

Immediately after setting the heater temperature or after $24 \mathrm{~h}$, depending on the particular test, hydration started. In some of the tests (FQ1/2, HI1/2, FQ2 and CG3) the water intake was also mea- 


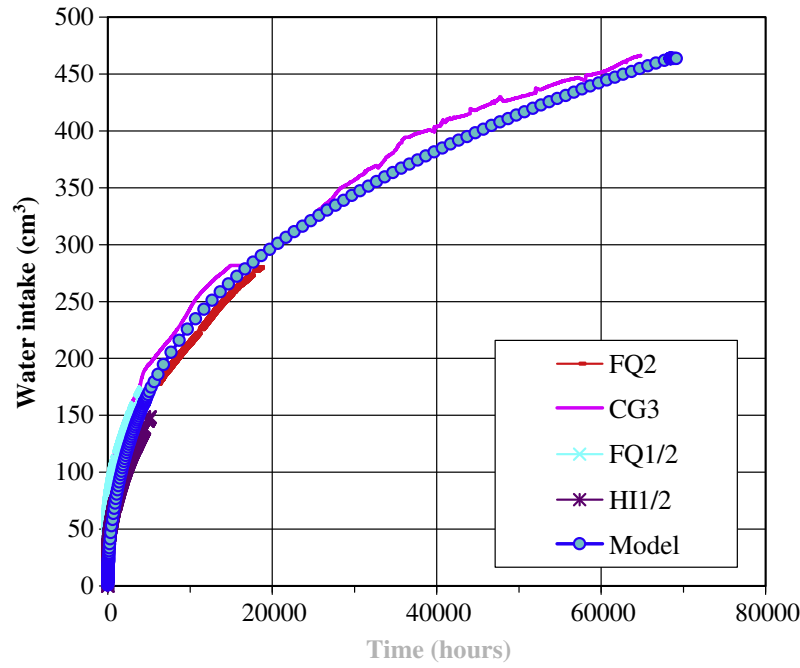

Fig. 8. Water intake during different duration tests: experimental and modelling results.

sured online. The water intake curves (Fig. 8), as measured by the volume change apparatuses, show disparity at the beginning of the tests, but tend to coincide as hydration progresses. The initial discrepancy is particularly clear for the two shortest tests, and can be mostiy attributed to the different roon operation of both tests: test FQ1/2 and test HI1/2 in autumn/winter, for $w$ take was higher in the first test, since t meability. It can be observed that to the hydraulic problem are quite gc vell captured by the model.

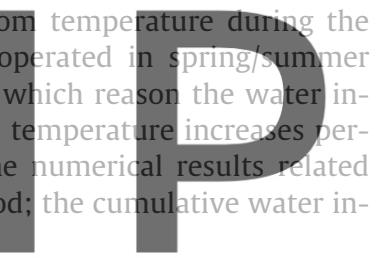

5.2. Postmortem measurements

Register for free at https//www.scipedia.com to

After switching off the heater and stopping the water injection, the cells were disassembled and the column of bentonite was extracted and weighed. In the longest test, CG3, the six bentonite blocks were sealed among them, although upon desiccation the two lower blocks could be detached. However, in the 1- and 2-year tests only the two upper blocks were sealed, i.e. the joint placed at $10 \mathrm{~cm}$ from the hydration surface, and in the 0.5 -year tests no joint was sealed. Shrinkage cracks near the heater were not observed in any of the tests.

The swelling of the hydrated bentonite might have deformed the Teflon walls of the cell, what would be confirmed by the higher difficulty of extracting from the cells the upper, most hydrated blocks. In fact, the diameter of the bentonite column at the end of test CG3 was not constant along it, and it overall had increased with respect to the initial diameter of $7.03 \mathrm{~cm}$. The inner diameter of the Teflon cell measured after disassembling and cooling ranged from $7.28 \mathrm{~cm}$ near the hydration surface to $7.05 \mathrm{~cm}$ near the heater, with an average value of $7.18 \mathrm{~cm}$. This increase is due to the deformation of the Teflon walls caused by the swelling of the bentonite upon saturation, which also implied an overall decrease of the dry density of the bentonite during the test to a value of $1.57 \mathrm{~g} / \mathrm{cm}^{3}$, according to these diameter measurements. Since the saturation of the bentonite in the shortest test was lower, it is expected that its overall swelling, and consequently its deformation and density decrease, was less important.

After slicing the columns, water content and dry density of each section were determined in two or three subsamples for most of the sections, although in some cases only one determination per section could be done. The distribution of water content along

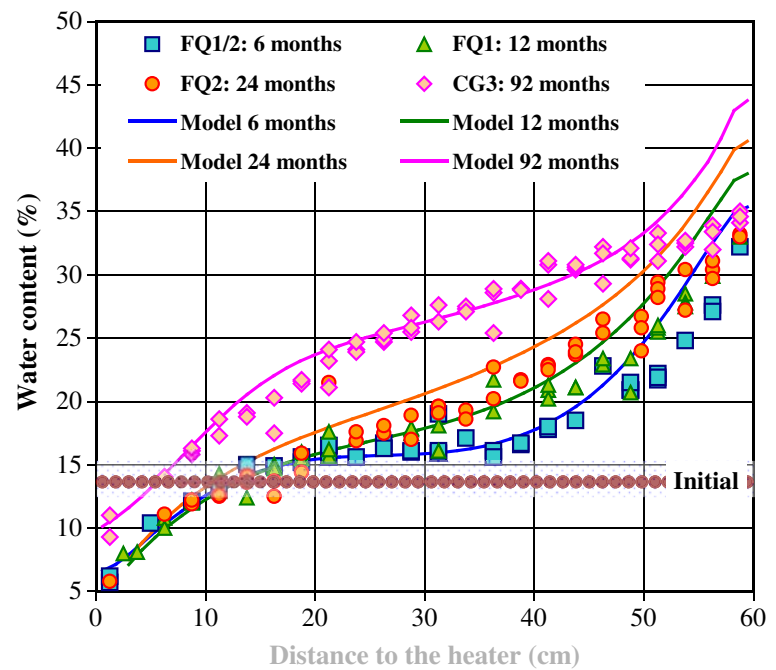

Fig. 9. Final water contents along the bentonite columns of the different tests: experimental and modelling results.

the bentonite column in the TH tests (Fig. 9), where an important gradient can be observed even in the longest test, shows the water content increased within the closest $20 \mathrm{~cm}$ from the hydration surface in the 6-month test, and within $40 \mathrm{~cm}$ in the 12 - and 24 month tests. In the 7.6-year test the increase of water confent took
place all over the column except in the $10 \mathrm{~cm}$ closest to the heater.
The water content reduction by the temperature effect is similar
for the three shorter durations, which means that desiccation takes
place rather quickly and only affects the closest $18 \mathrm{~cm}$ to the hea-
ter, where the water contents are 10 wer than the initial ones, which
were around $14 \%$. After 6 months, the clasest $18 \mathrm{gm}$ to the heater experienced a desiccation that is not recovered after 24 months; hence the water content remained in this zone below the initial va-

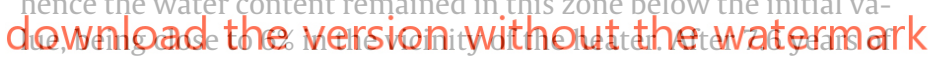
testing the water content of the bentonite is lower than the initial one only in the $5 \mathrm{~cm}$ closest to the heater.

The dry densities gradient along the bentonite column at the end of the tests (Fig. 10) reflects the different swelling of the bentonite, since the more hydrated sections have swollen more. The dry density decreases from the heater towards the hydration surface following an approximately linear trend. In the zones affected by hydration, the densities decreased below the initial value (nominal $1.65 \mathrm{~g} / \mathrm{cm}^{3}$ ) due to the expansion caused by saturation. On the contrary, near the heater, the dry density increased, due to the shrinkage caused by desiccation. Despite the dispersion of the data - caused by the initial inhomogeneities and by the determination method, it can be observed that the densities reached near the hydration surface were lower as the duration of the test increased (values as low as $1.41 \mathrm{~g} / \mathrm{cm}^{3}$ in test CG3), due to the fact that their saturation and, consequently, their swelling, was higher. Due to this density decrease, the degrees of saturation were lower than what could be expected by the high water content. In fact, degrees of saturation higher than $90 \%$ were only reached in the $3 \mathrm{~cm}$ closest to the hydration surface after 6 months of TH treatment, in $8 \mathrm{~cm}$ after 12 months, in $10 \mathrm{~cm}$ after 24 months and in $25 \mathrm{~cm}$ in the 7.6-year test. Taking a value of $1 \mathrm{~g} / \mathrm{cm}^{3}$ for the density of the adsorbed water, the $10 \mathrm{~cm}$ of bentonite closest to the hydration surface in test CG3 would be fully saturated. On the other hand, near the heater $(18 \mathrm{~cm})$ the densities remain the same from 6 to 24 months, and the degrees of saturation decrease from the initial $55-60 \%$ to values around $25 \%$ in the vicinity of the heater.

There is also an overall decrease of the average density of the bentonite, which is much more significant in the 7.6-year test. This 


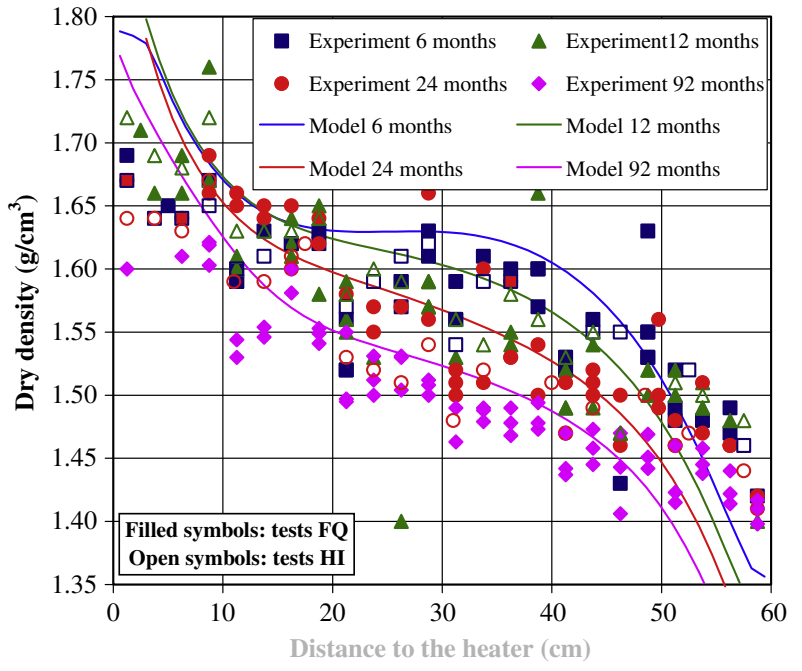

Fig. 10. Final dry densities along the bentonite columns of the different tests: experimental and modelling results.

is attributed to the deformation of the Teflon walls of the cell caused by the swelling of the bentonite, which increased over time, and also to the further expansion of the bentonite on dismantling of the cells and to the trimming of subsamples, which may cause

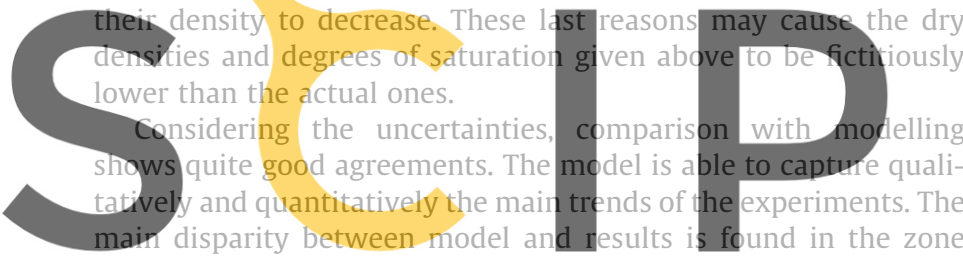
closest to the hydration front, especially in the longest tests. This can be caused by the unavoidable drying during sampling of the

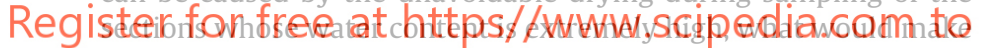
the water content and dry density measured lower and higher, respectively, than the actual ones.

Thus, the modelling of the heating and hydration of the cells has demonstrated the capability of the formulation and computer code to model the complex behaviour of expansive clays submitted to coupled THM actions.

\subsection{Discussion about final water content}

As it has been explained in the previous sections, the water intake at the end of the tests can be deduced from three different measurements. The most direct ones are the online measurement performed during the tests by the volume change apparatuses, and the weighing of the column at the end of the tests, that allows computing the water mass taken by comparison with the initial weight of the columns. Both methods are subject to error. The first one because the equipments used to measure online during such long periods of time can suffer failures and leaks, and the second one, because the extraction of the bentonite from the cells is difficult and some material can be lost during the process. This is the reason why there are not final weight values for tests FQ1/2 and HI1. An additional method to determine the water intake would be to compute it from the average water content measured in the different sections (Fig. 9).

The water content at the end of the tests according to the different measurements is shown in Table 2. A comparison of the values obtained with the three methods is given in Fig. 11. Despite several problems, due to various failures of the equipments, the final water contents calculated from the online water intake
Table 2

Final water intake and water content according to different measurements.

\begin{tabular}{lllllll}
\hline Test & $\begin{array}{l}\text { Duration } \\
(\text { days })\end{array}$ & $\begin{array}{l}\text { Water intake }^{\mathrm{a}} \\
\left(\mathrm{cm}^{3}\right)\end{array}$ & $\begin{array}{l}\text { Final } \\
w^{\mathrm{a}}(\%)\end{array}$ & $\begin{array}{l}\text { Water } \\
\text { intake }^{\mathrm{b}}(\mathrm{g})\end{array}$ & $\begin{array}{l}\text { Final } \\
w^{\mathrm{b}}(\%)\end{array}$ & $\begin{array}{l}\text { Final } \\
w^{\mathrm{c}}(\%)\end{array}$ \\
\hline FQ1/2 & 188 & 171 & 17.7 & & & 17.6 \\
$\mathrm{HI} 1 / 2$ & 214 & 128 & 17.3 & 116 & 17.0 & 16.9 \\
FQ1 & 370 & & & 165 & 18.2 & 18.5 \\
FQ2 & 762 & 280 & 20.6 & 235 & 19.4 & 20.2 \\
HI2 & & & 25.9 & 222 & 19.5 & \\
CG3 & 2775 & 461 & 256 & 24.7 & 25.0 \\
\hline
\end{tabular}

a Deduced from the water intake online measurements.

b Deduced from the difference between final and initial bentonite weight.

c Deduced from the average of the water content measured in the different sections.
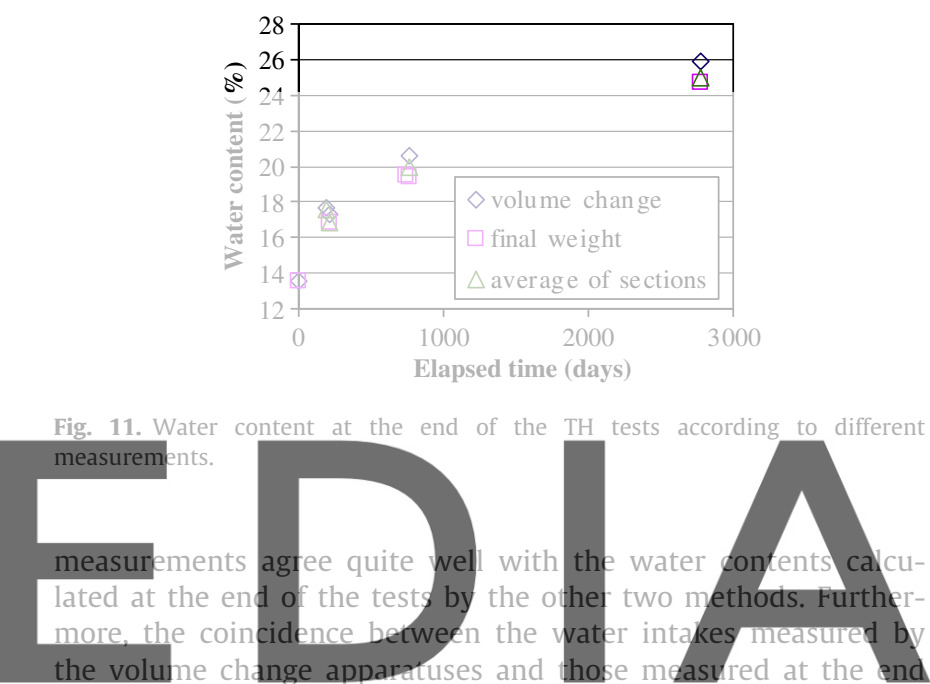

of the tests by final and initial weight differences and those determined by direct measurement in the different sections, allows to

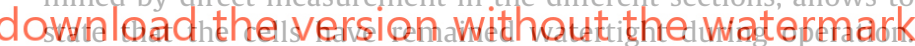
The coherence among values gives confidence on the results obtained and allows determining their possible range of variation. In addition, the agreement between model results and measurements is quite good.

\section{Summary and conclusions}

The conditions of the bentonite in an engineered barrier for HLW disposal have been simulated experimentally in a series of tests. Columns of compacted bentonite kept in hermetic Teflon cells were hydrated with low-salinity granitic water at the upper surface while they were heated at the bottom surface at a temperature of $100^{\circ} \mathrm{C}$ during different periods of time. The columns consisted of six stacked compacted blocks of $7 \mathrm{~cm}$ in diameter and $10 \mathrm{~cm}$ high. Consequently, the total length of clay inside the cells was $60 \mathrm{~cm}$, the same as the thickness of the bentonite barrier in the mock-up test of the FEBEX Project (ENRESA, 2006). The initial average dry density of the bentonite was $1.66 \mathrm{~g} / \mathrm{cm}^{3}$ and the water content $13.6 \%$. The duration of the tests was $6,12,24$ and 92 months. The temperatures inside the clay and the water intake were satisfactorily measured during the tests and, at the end, the cells were dismantled and the dry density and water content were determined in different positions along the blocks. The online measurements and the results of the postmortem tests performed in the bentonite after dismantling have been reported in the paper. In addition, the tests have been modelled as a boundary value problem using a numerical code especially developed to handle coupled THM problems in porous media, and the model results 
have been compared with the experimental measurements. The following conclusions can be drawn:

- There is a sharp temperature gradient in the vicinity of the heater. The temperatures inside the clay during the $\mathrm{TH}$ treatment are slightly lower as the duration of the test is longer, what is due to the increase in thermal conductivity of the clay with water content and the higher heat dissipation of the clay as it becomes wetter.

- At the end of all the tests there were important water content and dry density gradients along the bentonite column. The increase in water content caused by hydration is linked to a reduction in dry density as a consequence of swelling, whereas the decrease of water content caused by evaporation near the heater is linked to an increase of dry density as a consequence of shrinkage.

- The density and water content gradients will condition temporarily the hydro-mechanical properties of the bentonite that are dependent on both, as the permeability and the swelling capacity (Villar et al., 2005a, 2008).

Hydration and heating caused also geochemical modifications that are described elsewhere (Villar et al., 2008), in particular dissolution of some mineral species of the bentonite and movement of soluble ions by advection. The movement of some ions was affected by mineral equilibrium reactions (dissolution/precipitation) and exchange reactions, what gave place also to modification of the exchangeable cation


content is close to saturation.

The numerical model used can reproduce the global trends

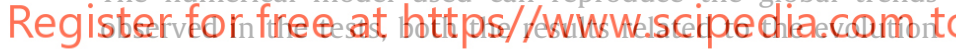
of the main variables of the tests and those related to the postmortem study. Most of the constitutive model and parameters assumed in the analysis presented here were developed and obtained from laboratory tests carried out during the FEBEX project and they are the same ones adopted for the main analysis of the large-scale heating tests of the FEBEX project (i.e. mock-up and in situ tests, ENRESA, 2006). The possibility to model properly heating tests at different scales with a unique set of parameters points to an appropriate selection of the processes and parameters.

- Indeed, there is still room for improvements and further developments are required. However, it is believed that the formulation provides a suitable platform for further advances and enhanced understanding. In fact, the consideration of the interactions between the two basic pore levels (macro and micropores) that exist in expansive clay was later on included in the model (Lloret et al., 2003), as well as the possible effect of the existence of a threshold hydraulic gradient on the hydraulic response of the barrier (Sánchez et al., 2007b) and of the impact of temperature on the hydro-mechanical properties of the bentonite (Sánchez et al., 2007a).

- Tests like those presented in this work, in which the expansive clay and the THM conditions are very similar to the ones expected in large-scale tests, are very useful for a better understanding of the behaviour of expansive clays under controlled thermal and hydraulic gradients similar to those expected in a HLW repository. This kind of experiments is also very useful for the validation of mathematical formulations and computer codes.

\section{Acknowledgments}

This research work has been carried out in the context of the FEBEX Project, financed by ENRESA (Spanish National Agency for Waste Management) and the European Commission (EC Contracts FI4W-CT95-006 and FIKW-CT-2000-00016). Test CG3 was finished and analysed in the framework of the NF-PRO Project, financed by ENRESA and the European Commission (EC Contract FI6W-CT2003-02389). P.L. Martín, J. Cuevas and J.M. Barcala supported the design and maintenance of the thermo-hydraulic tests. R. Campos and J. Aroz performed the laboratory work.

\section{Appendix A. Appendix}

\section{A.1. Mechanical constitutive model}

The mechanical law adopted is the Barcelona Basic Model (BBM). This model extends the concept of critical state for saturated soils to the unsaturated conditions, including the dependence of yield surface on suction. Two stress variables are considered: the net stresses $\left(\sigma-P_{\mathrm{g}} \boldsymbol{m}\right)$ and capillary suction. Net stress is the excess of total stress over gas pressure. If full saturation is achieved, net mean stress becomes effective stress. For simplicity net stress will also be denoted by $\sigma$.

A thermoplastic constitutive law (Alonso et al., 1990; Gens, 1995) has been selected based on a generalised yield surface that depends not only on stresses but on temperature and suction as

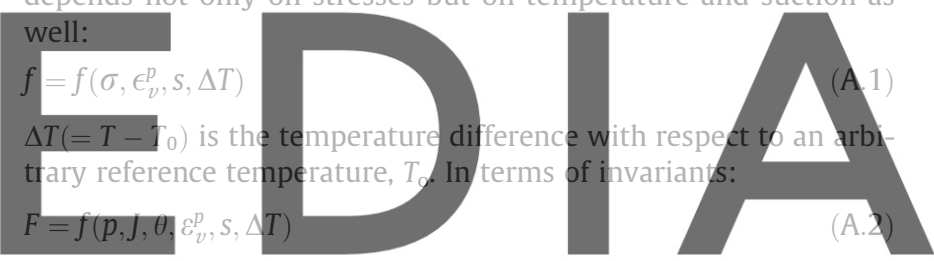

where

\section{dowinload thathe $y$ ersion without the watermark}

$J^{2}=1 / 2 \operatorname{trace}\left(s^{2}\right)$

$\theta=-\frac{1}{3} \sin ^{-1}\left(1.5 \sqrt{3} \operatorname{det} \mathbf{s} / J^{3}\right)$

$\mathbf{s}=\sigma-p \mathbf{I}$

I is the identity tensor. For simplicity, a form of the classical Modified Cam-clay model is taken as the reference isothermal saturated constitutive law:

$F=\frac{3 J^{2}}{g_{y}^{2}}-L_{y}^{2}\left(p+P_{s}\right)\left(P_{0}-P\right)=0$

Volumetric strain is defined as

$\varepsilon_{v}=\varepsilon_{x}+\varepsilon_{y}+\varepsilon_{z}$

and $\varepsilon_{v}^{p}$ is the plastic volumetric strain.

The basic assumption is that the pre-consolidation pressure, $P_{\mathrm{o}}$, is made dependent on both suction and temperature:

$P_{0}=P^{c}\left(\frac{P_{c}}{P^{c}}\right)^{\frac{\lambda(0)-k i o}{\lambda(s)-k i o}}$

$P_{c}=P_{0}^{*}+2\left(\alpha_{1} \Delta T+\alpha_{2} \Delta T(T)\right)$

$L_{y}=\frac{M}{g_{y}(\theta=-\pi / 6)}$

$\lambda(s)=\lambda(0)[(1-r) \exp (-\beta s)+r]$

In addition

$P_{s}=k \exp (-\rho \delta T) s$ 
In common with other critical state models, it is assumed that hardening depends on plastic volumetric strain only according to

$\dot{P}_{o}^{*}=\frac{1+e}{\lambda(0)-k_{i o}} P_{o}^{*} \dot{\varepsilon}_{v}^{p}$

and the plastic potential

$G=\frac{3 J^{2}}{g_{p}^{2}}-\alpha L_{p}^{2}\left(p+P_{s}\right)\left(P_{o}-p\right)$

$L_{p}=\frac{M}{g_{p}(\theta=-\pi / 6)}$

If $\alpha=1$, an associated plastic model results.

The variation of stress-stiffness with suction and, especially, the variation of swelling potential with stress and suction have been carefully considered. The resulting elastic model is as follows:

$\dot{\varepsilon}_{v}^{e}=\frac{k_{i}}{1+e} \frac{\dot{P}}{P}+\frac{k_{s}}{1+e} \frac{\dot{s}}{S+0.1}+\left(\alpha_{0}+2 \alpha_{2} \Delta T\right) \dot{T}$

$k_{i}=k_{i o}\left(1+\alpha_{i s} S\right)$

$k_{s}=k_{s 0}\left(1+\alpha_{s p} \ln p / P_{r}\right) \exp \left(\alpha_{s s} s\right.$

$\dot{E}=\dot{J} / G ; \quad G=E / 2(1+v)$

\section{References}

ENRESA, 2006. Full-scale Engineered Barriers Experiment. Updated Final Report 1994-2004. Publicación Técnica ENRESA 05-0/2006, Madrid, 590pp.

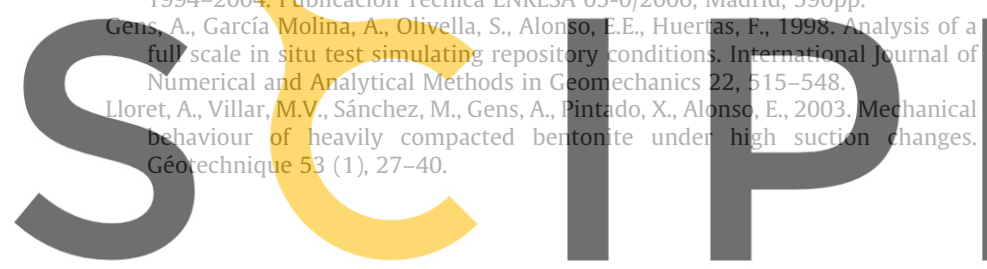

Lloret, A., Romero, E., Villar, M.V., 2004. FEBEX II Project Final report on thermohydro-mechanical laboratory tests. Publicación Técnica ENRESA 10/04, Madrid, 180pp.

Olivella, S., 1995. Non-isothermal multiphase flow of brine and gas through saline media. Ph.D. Thesis, Universitat Politècnica de Catalunya (UPC), Barcelona.

Olivella, S., Gens, A., 2000. Vapour transport in low permeability unsaturated soils with capillary effects. Transport in Porous Media 40, 219-241.

Olivella, S., Gens, A., Carrera, J., Alonso, E.E., 1994. Nonisothermal multiphase flow of brine and gas through saline media. Transport in Porous Media 15, 271-293.

Olivella, S., Gens, A., Carrera, J., Alonso, E.E., 1996. Numerical formulation for a simulator (CODE-BRIGHT) for the coupled analysis of saline media. Engineering Computations 13 (7), 87-112.

Pintado, X., Ledesma, A., Lloret, A., 2002. Backanalysis of thermohydraulic bentonite properties from laboratory tests. Engineering Geology 64, 91-115.

Sánchez, M., Gens, A., 2006. FEBEX Project final report. Final report on thermohydro-mechanical modelling. Publicación Técnica ENRESA 05-2/2006, Madrid, $163 p p$.

Sánchez, M., Villar, M.V., Gens, A., Olivella, S., Guimarães, L., do, N., 2007a. Modelling the effect of temperature on unsaturated swelling clays. In: Proc Int Symp on Numerical Models in Geomechanics (NUMOG X), Rhodes, April 2007.

Sánchez, M., Villar, M.V., Lloret, A., Gens, A., 2007b. Analysis of the expansive clay hydration under low hydraulic gradient. In: Experimental Unsaturated Soil Mechanics. Springer Proceedings in Physics, vol. 112. Springer, Berlin, pp. 309318

Van Genuchten, M.T., 1978. Calculating the unsaturated hydraulic conductivity with a new closed-form analytical model. Water Resources Research 37 (11), 21-28.

Villar, M.V., 2002. Thermo-hydro-mechanical characterisation of a bentonite from Cabo de Gata. A study applied to the use of bentonite as sealing material in high level radioactive waste repositories. Publicación Técnica ENRESA 01/2002. Madrid, 258pp.

Villar, M.V., Martín, P.L., Barcala, J.M., 2005a. Modification of physical, mechanical and hydraulic properties of bentonite by thermo-hydraulic gradients. Engineering Geology 81, 284-297.

Villar, M.V., Sánchez, M., Lloret, A., Gens, A., Romero, E., 2005b. Experimental and

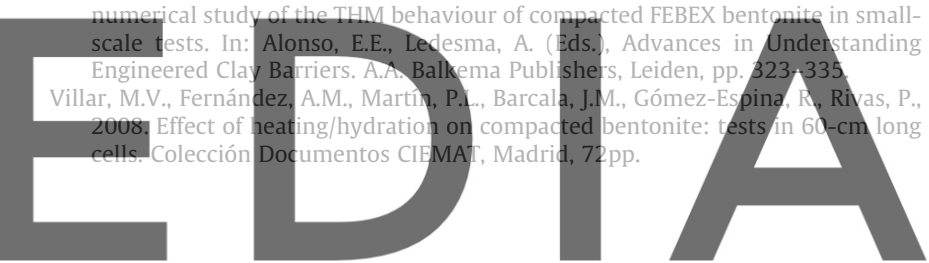

